\title{
A INFLUÊNCIA DA REALIDADE VIRTUAL SOBRE A VELOCIDADE DA MARCHA E AVALIAÇÃO DA SATISFAÇÃO DE INDIVÍDUOS COM DOENÇA DE PARKINSON
}

\author{
Mariana da Cruz Souza, Paola Larissa de Araujo Biazini, Débora Toshimi Furuta, Alice Haniuda Moliterno, \\ Nicoly Ribeiro Uliam, Dyenifer Fernandes de Oliveira, Isabella Cristina Leoci, Isabela Bortolim Frasson, Silas \\ de Oliveira Damasceno, Caroline Nunes Gonzaga, Guilherme Yassuyuki Tacao, Augusto Cesinando de \\ Carvalho.
}

Universidade Estadual Paulista Júlio De Mesquita Filho - UNESP, Presidente Prudenee, SP. E-mail: fisiomarianasouza@hotmail.com

\section{RESUMO}

A Realidade Virtual (RV) é uma ferramenta promissora utilizada na reabilitação de indivíduos com Doença de Parkinson (DP) pois promove aprendizagem motora pelo feedback multissensorial em tempo real. Tratase de um estudo experimental de braço único cujo objetivo foi analisar a influência da RV sobre a velocidade da marcha e satisfação de indivíduos com DP. Na avaliação inicial (AV1) aplicou-se Teste de Caminhada de 10 metros (TC10), seguido do protocolo de 10 sessões com RV utilizando Nintendo Wii ${ }^{\mathrm{TM}}$. Na avaliação final (AV2), aplicou-se novamente o TC10 seguido da Escala de Avaliação da Satisfação dos

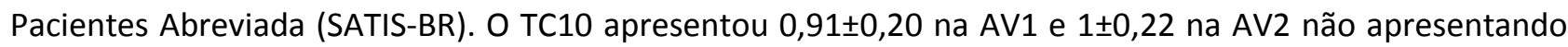
diferença significativa $(p=0,187)$ enquanto a SATIS-BR pontuou $4,8 \pm 0,22$ de um total de 5 . Conclui-se que apesar do resultado não significativo, a velocidade da marcha foi mantida, expondo valores expressivos da satisfação dos participantes.

Palavras-chave: doença de parkinson, realidade virtual, marcha, satisfação do paciente, fisioterapia

\section{THE INFLUENCE OF VIRTUAL REALITY ON GAIT SPEED AND SATISFACTION ASSESSMENT OF INDIVIDUALS WITH PARKINSON'S DISEASE}

\begin{abstract}
Virtual Reality (VR) is a promising tool used in the rehabilitation of individuals with Parkinson's Disease (PD) as it promotes motor learning through multisensory feedback in real time. This is an experimental study with a single arm whose objective was to analyze the influence of VR on gait speed and satisfaction assessment of individuals with PD. In the initial assessment (IA1) a 10-Meter Walk Test (10MWT) was applied, followed by the protocol of 10 sessions with VR using Nintendo $\mathrm{Wii}^{\mathrm{TM}}$ console. In the final evaluation (FE2), the 10MWT was reapplied, followed by the Abbreviated Patients' Satisfaction with Mental Health Services Scale (SATIS-BR). The 10MWT showed $0.91 \pm 0.20$ in IA1 and $1 \pm 0.22$ in FE2 with no significant difference ( $p=0.187$ ) while SATIS-BR scored $4.8 \pm 0.22$ out of 5 . It is concluded that despite the non-significant result, the gait speed was maintained, exposing expressive values of the participants' satisfaction.
\end{abstract}

Keywords: parkinson disease, virtual reality, gait, patient satisfaction, physical therapy

\section{INTRODUÇÃO}

A Realidade Virtual (RV) é uma ferramenta nova e promissora ${ }^{1,2}$ que surgiu como uma tecnologia auxiliadora ao processo de reabilitação em pacientes com doenças neurológicas ${ }^{3}$. Tem se tornado uma alternativa valiosa às abordagens convencionais ${ }^{1}$ pois busca promover a aprendizagem motora pelo feedback multissensorial em tempo real ${ }^{1,2,3,4,5}$. 
O ambiente de RV, por se tratar de práticas de tarefas complexas ${ }^{3,6}$, em vez da repetição de um movimento simples é capaz de induzir a reorganização da arquitetura neural e promover a neuroplasticidade, estimulando tanto a recuperação das habilidades motoras ${ }^{3,7}$ e cognitivas quanto função executiva e memória ${ }^{8}$, o que leva ao estudo de diferentes alternativas de terapias para doenças neurológicas.

Dentre as doenças de ordem neurológica, a Doença de Parkinson (DP) é uma das mais comuns em todo o mundo ${ }^{1,9,10}$. Caracteriza-se por uma condição crônica, degenerativa e progressiva do sistema nervoso central que causa deficiências, tanto motoras quanto cognitivas.

A alteração da marcha é uma das principais características da DP. Normalmente os sujeitos caminham lentamente com passos pequenos e arrastados, apresentam diminuição do balanço do braço e postura flexionada para frente. Esses comportamentos e características, podem causar redução da velocidade da caminhada, diminuição do comprimento da passada e aumento da cadência ${ }^{10-13}$. Em casos mais avançados, apresentam marcha congelada, na qual, apesar da tentativa do paciente de andar, a progressão dos pés para frente é significativamente reduzida, aumentando o risco de quedas $\mathrm{s}^{1,2,10,12,14,15}$. Podem apresentar também fadiga, apatia, depressão e ansiedade afetando substancialmente o funcionamento e a qualidade de vida do paciente ${ }^{1,10}$.

Atualmente, os medicamentos que visam reduzir o congelamento da marcha na DP não fornecem ao paciente uma resposta totalmente eficaz. Há fortes evidências de que a fisioterapia por meio de diferentes estratégias pode melhorar as funções motoras e cognitivas de indivíduos com $\mathrm{DP}^{6,7,2}$ pois apresentam benefícios significativos na velocidade da marcha, comprimento do passo, caminhada, giro, equilíbrio global e deficiências motoras ${ }^{1,10,15}$.

Embora estudos recentes em DP tenham demonstrado que o exercício prolongado foi capaz de promover benefícios motores e cognitivos, envolver os pacientes em programas de exercícios regulares de longo prazo é desafiador ${ }^{1}$. A fisioterapia convencional é por sua natureza repetitiva e a repetição tende a reduzir a motivação e aderência do paciente ao tratamento $^{14}$.

Para superar essa monotonia no desempenho das tarefas, os avanços tecnológicos vem sendo gradativamente incorporados com 0 Colloq Vitae 2020 set-dez; 12(3): 1-9.

Artigo Open Access sob uma licença CC BY-NC-ND (http://creativecommons.org/licenses/by-nc-nd/4.0/). objetivo de melhorar a qualidade de vida das pessoas $^{14}$. Na última década, os dispositivos de jogos comerciais como Nintendo Wii e Microsoft Kinect se tornaram uma ferramenta em RV no processo de reabilitação devido ao seu baixo custo em comparação à outros métodos de tratamento $4,5,6,14,16,17$. Além disso, é capaz de facilitar a realização de exercícios de alto volume e melhorar o controle postural, mobilidade, desempenho da marcha e do equilíbrio em pessoas com $\mathrm{DP}^{2,10}$.

Os videogames comerciais, apesar de não terem sido desenvolvidos especificamente para indivíduos com distúrbios neurológicos, são capazes de estimular deslocamentos multidirecionais, transferência de peso, elevado número de repetições, planejamento e tomada de decisão, além da concentração sustentada que é necessária promovendo motivação e comprometimento nas tarefas realizadas.

A RV tem também um papel muito positivo na motivação e aderência dos participantes ${ }^{1,5}$, uma vez que sua interface replica cenários da vida real ${ }^{4,1}$ oferecendo um maior potencial de transferência para atividades funcionais da vida diária ${ }^{1}$. Torna-se, então, uma maneira personalizada e motivadora ${ }^{1,4}$, que traz diversão e prazer ao processo de recuperação $0^{1,2,3,4}$.

É importante mensurar os resultados e as interferências no tratamento para guiar e aprimorar cada vez mais a conduta terapêutica, para isso, é fundamental uma avaliação efetiva com testes, escalas e questionários.

$\mathrm{O}$ estudo teve por objetivo aplicar um protocolo de jogos utilizando Nintendo Wii e investigar os possíveis efeitos sobre a marcha e satisfação de indivíduos com DP, a fim de que se possa analisar a efetividade da RV como forma de tratamento para essa população.

\section{MÉTODOS \\ PARTICIPANTES E CRITÉRIOS DE ELEGIBILIDADE}

Foram selecionados para participar do estudo indivíduos com DP assistidos por um serviço de Fisioterapia e Reabilitação de uma clínica escola na cidade de Presidente PrudenteSP. Trata-se de um estudo clínico experimental, longitudinal, prospectivo e de braço único. Os participantes foram informados sobre os procedimentos adotados e os respectivos objetivos da pesquisa, e após esclarecimentos assinaram o Termo de Consentimento Livre e Esclarecido (TCLE), previamente aprovado pelo 
Comitê de Ética da FCT-UNESP (CAAE: 15220619.0.0000.5402). Os procedimentos adotados nesta pesquisa obedecem aos Critérios da Ética em Pesquisa com Seres Humanos conforme resolução no. 466/2012 do Conselho Nacional de Saúde. Para serem incluídos na pesquisa, os participantes precisavam apresentar DP, com encaminhamento médico e classificação do estágio da lesão pela escala Hoehn e Yahr, a qual varia de $0-5$ (onde $0=0$ indivíduo não apresenta sintomas de doença, e $5=$ apresenta sintomas bilateralmente; incapacidade de andar) $)^{11}$, sendo capaz de realizar deambulação ativa (com ou sem dispositivo auxiliar de locomoção e órteses), além de ausência de déficits cognitivos avaliados pelo instrumento Mini Exame do Estado Mental ${ }^{18}$ (considerando o nível de escolaridade ${ }^{19}$ - sendo a nota de corte para população analfabeta $18 / 19$ e para a população com instrução escolar $24 / 25)^{20}$. Foram excluídos participantes que relatassem outras doenças neurológicas e/ou circunstâncias e dificuldades que impedissem a realização de algum procedimento do estudo.

\section{DESENHO DO ESTUDO}

Inicialmente foi realizada uma entrevista individual para coleta de dados pessoais e verificação dos critérios de inclusão, em seguida foi realizada uma avaliação inicial (AV1) utilizando o Teste de Caminhada de $10 \mathrm{~m}$ (TC10) para a avaliação dos comprometimentos motores. Após 10 sessões de intervenção utilizando RV, foi realizada uma segunda avaliação (AV2) aplicando TC10 novamente, e a seguir a Escala de Avaliação da Satisfação dos Pacientes Abreviada (SATIS-BR) para a análise dos aspectos biopsicossociais.

\section{INSTRUMENTOS DE AVALIAÇÃO TESTE DE CAMINHADA DE 10 METROS (TC10)}

O Teste de Caminhada de 10 metros (TC10) avalia a velocidade da marcha do indivíduo. $\mathrm{O}$ mesmo consiste em caminhar numa velocidade costumeira em um corredor de no mínimo 14 metros, onde se é registrado com um cronômetro o tempo em que se percorre os 10 metros centrais (desconsiderando os 2 metros iniciais e finais). Para classificação, os participantes foram identificados conforme a velocidade de deambulação, sendo separadas em três classes: marcha domiciliar $(<0,4 \mathrm{~m} / \mathrm{s})$, marcha comunitária limitada $(0,4$ a $0,8 \mathrm{~m} / \mathrm{s})$, e marcha comunitária $(>0,8 \mathrm{~m} / \mathrm{s})^{21}$.

\section{ESCALA DE AVALIAÇÃO DA SATISFAÇÃO DOS PACIENTES ABREVIADA (SATIS-BR)}

A versão abreviada da SATIS-BR trata-se de uma escala que contém 15 questões, unidas em subescalas que propõe avaliar a satisfação dos pacientes com a equipe e com o serviço prestado. Dessas, 3 perguntas são qualitativas complementares a escala global relacionado a percepção sobre as informações do serviço ofertado. Para cada resposta, a escala do tipo Likert com 5 pontos foi utilizada, onde 1= muito insatisfeito e $5=$ muito satisfeito ${ }^{22,23}$.

\section{PROTOCOLO DE REALIDADE VIRTUAL}

Os participantes foram submetidos, individualmente, ao atendimento fisioterapêutico com a RV não imersiva. O protocolo de jogos com RV foi realizado utilizando o console Nintendo $\mathrm{Wii}^{\mathrm{TM}}$ (desenvolvedor Nintendo e fabricante Foxconn) sendo utilizados 6 jogos do Wii Fit ${ }^{\text {TM }}$ Plus e Wii Sports Resort ${ }^{T M}$ (especificados na Tabela 1) com os acessórios Wii Balance Board (plataforma) e Wii Remote (controle). As sessões duravam aproximadamente 45 minutos cada, sendo realizadas duas sessões por semana, totalizando dez sessões.

O participante se posicionava em pé, em frente a uma televisão à uma distância de 150 centímetros. Inicialmente, os participantes realizaram uma sessão piloto a fim de conhecerem o dispositivo, jogos e se familiarizar com a terapia com RV. No início e no fim de cada sessão, a pressão arterial e frequência cardíaca eram aferidas como forma de monitoramento. 
Tabela 1. Descrição dos jogos

\begin{tabular}{ll}
\hline JOGOS & \multicolumn{1}{c}{ DESCRIÇÃO } \\
\hline Free Run & $\begin{array}{l}\text { Caracteriza-se por realizar uma caminhada estática em velocidade } \\
\text { constante com duração de } 5 \text { minutos como forma de aquecimento; }\end{array}$ \\
\hline Hula Hoop & $\begin{array}{l}\text { Realizam-se movimentos circulares com o quadril sobre a plataforma Wii } \\
\text { Balance Board, simulando o domínio de um bambolê sem deixá-lo cair; }\end{array}$ \\
\hline Perfect 10 & $\begin{array}{l}\text { Realizam-se movimentos com o quadril para acertar cogumelos, sem } \\
\text { mover os pés do lugar sobre a plataforma Wii Balance Board. Ao mesmo } \\
\text { tempo em que se realizam contas matemáticas; }\end{array}$ \\
\hline Penguin Slide & $\begin{array}{l}\text { Representado por um pinguim sobre a plataforma Wii Balance Board, } \\
\text { representada no jogo por uma plataforma de gelo, o participante deve } \\
\text { realizar leves deslocamentos de peso para ambos os lados com o objetivo } \\
\text { de se alimentar dos peixes; }\end{array}$ \\
\hline Free Step & $\begin{array}{l}\text { Consiste em subir e descer da plataforma Wii Balance Board, que } \\
\text { representa um step, mantendo o ritmo constante e alternando os pés a } \\
\text { cada minuto }\end{array}$ \\
\hline Table Tennis & $\begin{array}{l}\text { Simulador de tênis de mesa, no qual o controle Wii Remote representa a } \\
\text { raquete. }\end{array}$
\end{tabular}

\section{ANÁLISE ESTATÍSTICA}

Após a tabulação dos dados dispostos em planilha pré-definida no software Microsoft Office Excel, a distribuição normal foi testada pelo teste de Shapiro Wilk. Para comparação das escalas foi utilizado o teste T-student para amostras pareadas. Os dados expressos em média e desvio padrão foram rodados pelo programa GraphPad Prism. Considerou-se significante o valor de $p<0,05$.

\section{RESULTADOS}

Fizeram parte do estudo 7 indivíduos com $D P$, sendo 6 do gênero feminino e 1 do gênero masculino, apresentando idade média de
$69,71 \pm 7,20$ anos e classificação média da escala Hoehn e Yahr modificada de 2,35 $\pm 0,47$. A média apontada no Mini-exame do Estado Mental foi de $28,28 \pm 0,95$ pontos, sendo a média de escolaridade de $9,86 \pm 4,56$ anos.

Os resultados obtidos no TC10 foram $0,91 \pm 0,20$ na $A V 1$ e $1 \pm 0,22$ na $A V 2$, não demonstrando diferença estatística significativa $(p=0,187)$. Contudo, nota-se na Figura 1 que $71,42 \%$ dos participantes apresentaram melhora na velocidade da marcha na AV2 em relação a $\mathrm{AV} 1$, além de $28 \%$ passar de marcha comunitária limitante $(0,4$ e $0,8 \mathrm{~m} / \mathrm{s})$ para marcha comunitária $(>0,8 \mathrm{~m} / \mathrm{s})$.

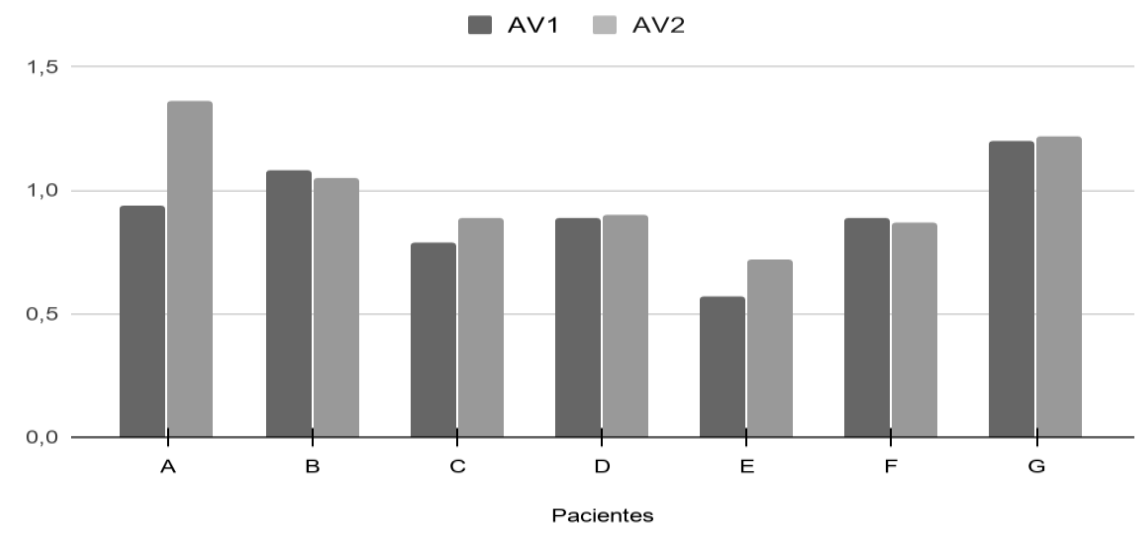

Figura 1. Valores individuais obtidos no TC10. Legenda: $A V 1$ = avaliação inicial; $A V 2$ = avaliação final; Pacientes representados de A - G. 
Os valores médios obtidos na SATIS-BR

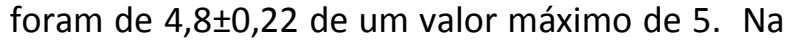
tabela 2 abaixo pode-se observar os valores médios obtidos das três subescalas que a compõem, sendo consideradas a satisfação com a equipe, satisfação com o serviço e percepção sobre os diversos aspectos dos serviços oferecidos. Os resultados obtidos demonstraram alto nível de satisfação dos participantes visto que apresentaram valores próximos a classificação muito satisfeito (nota 5), com destaque na satisfação com o serviço, no qual obteve $100 \%$ de satisfação.

Tabela 2. Resultados comparativos das subescalas que compõem a SATIS-BR.

\begin{tabular}{lc}
\hline SUBESCALAS & MÉDIA/DP \\
\hline Satisfação com a equipe & $4,70 \pm 0,26$ \\
\hline Satisfação com o serviço & 5 \\
\hline Percepção sobre os diversos aspectos dos serviços oferecidos & $4,7 \pm 0,39$
\end{tabular}

Legenda: SATIS-BR = Escala de Avaliação da Satisfação dos pacientes Abreviada; DP= Desvio Padrão

\section{DISCUSSÃO}

Este estudo avaliou o efeito da RV sobre a velocidade da marcha e a satisfação de indivíduos com DP por meio dos jogos do Nintendo $\mathrm{Wii}^{\mathrm{TM}}$. Os resultados obtidos no TC10 não apresentou melhoras significativas na velocidade da marcha, entretanto, os indivíduos submetidos à terapia com RV apresentaram um alto nível de satisfação quanto a equipe, serviços e diversos aspectos dos serviços oferecidos.

Os resultados mostraram que a RV não apresentou melhoras significativas na velocidade da marcha pelo TC10 o que corrobora os resultados obtidos na revisão sistemática de Wang et $a l^{24}$, que ao comparar RV com terapia convencional, utilizando o TC10 como uma das avaliações pós intervenção, não obteve melhora estatística significante na velocidade da marcha dos indivíduos avaliados. A amostra deste estudo, apesar de não apresentar diferença estatística significante, permaneceu dentro do padrão, considerando que a média da velocidade da marcha em indivíduos com DP, estabelecida no estudo de Combs et al. ${ }^{13}$, está entre 0,18-1,21 $\mathrm{m} / \mathrm{s}$, sendo observada por Cano Porras $D$ et $\left.a\right|^{3}$ uma variação entre 10 e $14 \%$ apenas na melhora dos resultados por meio do TC10.

Rochester et $a l^{25}$, em estudo realizado com 153 pessoas com DP indicou que $70 \%$ dos pacientes realizavam marcha comunitária com velocidade de marcha de $0,88 \mathrm{~m} / \mathrm{s}$. Analisando individualmente $o$ desempenho dos participantes Colloq Vitae 2020 set-dez; 12(3): 1-9.

Artigo Open Access sob uma licença CC BY-NC-ND (http://creativecommons.org/licenses/by-nc-nd/4.0/). do presente estudo percebe-se que $71,42 \%$ apresentaram melhora na velocidade da marcha demonstradas na Figura 1 classificando-se em marcha comunitária $(>0,8 \mathrm{~m} / \mathrm{s})$ por Bowden MG et $a l^{21}$.

O grupo amostral se compôs de idosos com idade média de $69,71 \pm 7,20$ anos sendo $85,7 \%$ do gênero feminino, o que, segundo Rochester et $a l^{25}$ e Elbers et al. ${ }^{26}$ justificaria uma marcha mais lenta juntamente com outros fatores, como gravidade da doença, depressão ${ }^{25,26}$, ansiedade, fadiga e confiança no equilíbrio $^{26}$. A diferença entre a marcha confortável e a marcha rápida está altamente relacionada às quedas nos últimos 6 meses ${ }^{27}$. Nota-se, então, a importância da aplicação do TC10, visto que analisar a velocidade geral da marcha não está ligado apenas ao estado físico mas também social e psicológico ${ }^{26}$.

Para que os indivíduos com DP otimizem a velocidade de marcha, percebe-se que apesar de existirem fatores imutáveis como idade, sexo e gravidade da doença, existem fatores mutáveis como: manejo da ansiedade e depressão, aumento da confiança no equilíbrio que podem ser facilmente trabalhados. A RV é uma excelente alternativa podendo influenciar não só nos ganhos motores mas na melhora da memória e redução da ansiedade ${ }^{17}$. Ao dispor de um feedback multissensorial em tempo real onde o participante se vê representado por um avatar dentro do jogo, a RV se torna um desafio, em 
que, a cada sessão ao superar sua pontuação anterior, intensifica seu esforço e número de repetições, indispensável para o desenvolvimento da neuroplasticidade. Segundo Alves et al. ${ }^{17}$, é capaz de reduzir, de trabalhar a ansiedade, muito prevalente e impactante nas atividades de vida diária e na qualidade de vida dos pacientes com $\mathrm{DP}^{28}$.

O protocolo de RV aplicado no presente estudo totalizou 10 sessões que foram aplicadas 2 vezes por semana, com duração de 45 minutos cada, em um período de 5 semanas aproximadamente. Estudo este semelhante ao de Alves et al. ${ }^{17}$ que realizou um ensaio clínico totalizando 10 sessões de $45-60$ minutos, durante 5 semanas, e que assim como este estudo, também não apresentou melhoras significativas na velocidade da marcha. O que leva a uma interpretação de que o tempo e período de sessões, ou até mesmo a forma como o estímulo é oferecido aos pacientes, pode influenciar em resultados não significativos.

Em contrapartida Zettergren et al. $^{29} \mathrm{e}$ Gonçalves GB et al. ${ }^{30}$ testaram um volume maior de sessões e obtiveram melhores resultados. Zettergren et $a .^{29}$ realizou um estudo de caso totalizando 16 sessões, que foram aplicadas 2 vezes por semana por um período de 8 semanas, e observou melhora no desempenho funcional e maior velocidade de marcha. Já no estudo de Gonçalves GB et al. $^{30}$ foram um total de 14 sessões aplicadas 2 vezes por semana, com duração de 40 minutos cada. Isso traz a possibilidade de que 10 sessões seja um volume baixo para a obtenção de ganhos na marcha sendo necessário um aumento no número de sessões e até mesmo melhora no estímulo que é oferecido.

Muitos estudos mostram que a RV pode ser uma boa ferramenta quando aliada à terapia convencional na $D P^{13}$. O Nintendo Wii é uma ótima alternativa de reabilitação motora em indivíduos pois auxilia na execução de técnicas e atividades mais funcionais, que seriam de difícil realização dentro de um ambiente clínico e com aparelhos convencionais. Os dispositivos auxiliares como Wii Balance Board e Wii Remote promovem uma variedade de possibilidades terapêuticas que promovem a descarga de peso, equilíbrio e controle motor. Além de ser uma tecnologia lúdica e divertida que promove maior adesão dos participantes.

Visto que a RV é uma ferramenta atual e a amostra se constituiu de pacientes idosos, a Colloq Vitae 2020 set-dez; 12(3): 1-9.

Artigo Open Access sob uma licença CC BY-NC-ND (http://creativecommons.org/licenses/by-nc-nd/4.0/). intervenção proposta, além dos benefícios terapêuticos propõe também uma aproximação entre gerações distintas, incluindo esse indivíduo na era da tecnologia.

A satisfação geral dos participantes foi notável, com destaque na subescala de satisfação com o serviço, a qual obteve-se pontuação de $100 \%$. Este dado, quando comparado ao valor obtido no TC10, nos mostra um fator importante, pois mesmo os pacientes não tendo apresentado melhoras significativas na velocidade da marcha, todos se mostraram $100 \%$ satisfeitos com o serviço prestado.

Na análise da satisfação da subescala da equipe, nota-se que a pontuação obtida foi alta, sendo $4,70 \pm 0,26$ de uma pontuação máxima de 5 , o que reforça o fato de que a equipe de fisioterapeutas no processo de reabilitação de indivíduos com DP é de extrema importância. Não diz respeito apenas à terapia proposta, mas em enxergar o indivíduo como um todo e não somente sua doença. Faz com que o participante se sinta respeitado e importante, o que por sua vez aumenta a aderência à terapia. Os indivíduos, ao procurarem os serviços de saúde, buscam ser ouvidos e entender o processo de sua patologia, assim como as condutas que serão adotadas para que estes se recuperem.

Para Kantorski et $a .^{31}$ o modo
psicossocial visa a promover um reposicionamento do sujeito, possibilitando que a pessoa se reconheça como um dos agentes implicados e se posicione como um agente de mudanças. Entretanto, Thiengo et al. ${ }^{32}$ salienta que ao se trabalhar com medidas de satisfação, podem ocorrer vieses que resultem em respostas excessivamente positivas, tais como o viés de gratidão e/ou de aceitação. Quando se trata de serviços públicos, é provável que os familiares se sintam constrangidos e relutantes em criticar os serviços sobre os quais eles são tão dependentes aliado ao medo de perder o acesso ao serviço.

Destaca-se como limitação deste estudo o baixo número amostral, baixo volume e/ou intensidade de sessões e a dificuldade de alguns participantes na execução dos jogos devido aos seus comprometimentos e/ou pouca familiarização com a nova terapêutica, o que pode ter contribuído para os resultados não expressivos. Sugere-se aos próximos estudos um maior tempo de familiarização dos participantes com a nova tecnologia, além de um possível número amostral maior com maior número de sessões. 
Embora não tenha apresentado mudanças significativas na velocidade da marcha em indivíduos com DP, obteve-se valores expressivos da satisfação dos voluntários indicando que a RV é uma boa intervenção terapêutica, capaz de motivar os pacientes. Conclui-se que este estudo apresenta relevância científica, pois a RV trouxe uma nova vertente terapêutica moderna, alternativa e muito promissora que deve ser melhor explorada.

\section{CONFLITO DE INTERESSES}

Os autores declararam não haver qualquer potencial conflito de interesse que possa interferir na imparcialidade deste trabalho científico.

\section{REFERÊNCIAS}

1. Dockx K, Bekkers EM, Van den Bergh V, Ginis P, Rochester L, Hausdorff JM et al. Virtual reality for rehabilitation in Parkinson's disease. Cochrane Database Syst Rev. 2016; 12(12):CD010760. https://doi.org/10.1002/14651858.cd010760.pub $\underline{2}$

2. Feng H, Li C, Liu J, Wang L, Ma J, Li G et al. Virtual Reality Rehabilitation Versus Conventional Physical Therapy for Improving Balance and Gait in Parkinson's Disease Patients: A Randomized Controlled Trial. Med Sci Monit. 2019 Jun 5; 25: 4186-92. https://doi.org/10.12659/MSM.916455

3. Cano Porras D, Siemonsma P, Inzelberg R, Zeilig $\mathrm{G}$, Plotnik M. Advantages of virtual reality in the rehabilitation of balance and gait: Systematic review. Neurology. 2018 May 29; 90(22):1017-25. http://doi.org/10.1212/WNL.0000000000005603

4. Perrochon A, Borel B, Istrate D, Compagnat $M$, Daviet JC. Exercise-based games interventions at home in individuals with a neurological disease: $A$ systematic review and meta-analysis. Ann Phys Rehabil Med. 2019 Sep;62(5):366-78. http://doi.org/10.1016/i.rehab.2019.04.004

5. Triegaardt J, Han TS, Sada C, Sharma S, Sharma $P$. The role of virtual reality on outcomes in rehabilitation of Parkinson's disease: metaanalysis and systematic review in 1031 participants. Neurol Sci. 2020 Mar; 41(3):529-36. http://doi.org/10.1007/s10072-019-04144-3

6. Silva KG, De Freitas TB, Doná F, Ganança FF, Ferraz HB, Torriani-Pasin C et al. Effects of virtual rehabilitation versus conventional physical Colloq Vitae 2020 set-dez; 12(3): 1-9.

Artigo Open Access sob uma licença CC BY-NC-ND (http://creativecommons.org/licenses/by-nc-nd/4.0/). therapy on postural control, gait, and cognition of patients with Parkinson's disease: study protocol for a randomized controlled feasibility trial. Pilot Feasibility Stud. 2017 Dec 6 ; 3: 68. http://doi.org/10.1186/s40814-017-0210-3

7. Galna B, Jackson D, Schofield G, McNaney R, Webster $M$, Barry $G$ et al. Retraining function in people with Parkinson's disease using the Microsoft kinect: game design and pilot testing. J Neuroeng Rehabil. 2014 Apr 14; 11:60. http://doi.org/10.1186/1743-0003-11-60

8. Costa MTS, Vieira LP, Barbosa EO, Mendes Oliveira L, Maillot P, Ottero Vaghetti CA et al. Virtual Reality-Based Exercise with Exergames as Medicine in Different Contexts: A Short Review. Clin Pract Epidemiol Ment Health. 2019 Jan 31; 15:15-20.

http://doi.org/10.2174/1745017901915010015

9. Pazzaglia C, Imbimbo I, Tranchita E, Minganti C, Ricciardi D, Lo Monaco R et al. Comparison of virtual reality rehabilitation and conventional rehabilitation in Parkinson's disease: a randomised controlled trial. Physiotherapy. 2020 Mar; 106:36-42.

http://doi.org/10.1016/j.physio.2019.12.007

10. Nuic $D$, Vinti $M$, Karachi $C$, Foulon $P$, Van Hamme A, Welter ML. The feasibility and positive effects of a customised videogame rehabilitation programme for freezing of gait and falls in Parkinson's disease patients: a pilot study. J Neuroeng Rehabil. 2018 Apr 10; 15(1):31. http://doi.org/10.1186/s12984-018-0375-x

11. Kobayashi E, Himuro N, Takahashi M. Clinical utility of the 6-min walk test for patients with moderate Parkinson's disease. Int J Rehabil Res. 2017 Mar; 40(1):66-70. http://doi.org/10.1097/MRR.0000000000000205

12. Mirelman A, Bonato P, Camicioli R, Ellis TD, Giladi N, Hamilton JL et al. Gait impairments in Parkinson's disease. Lancet Neurol. 2019 Jul; 18(7):697-708. http://doi.org/10.1016/S1474$\underline{4422(19) 30044-4}$

13. Combs SA, Diehl MD, Filip J, Long E. Shortdistance walking speed tests in people with Parkinson disease: reliability, responsiveness, and validity. Gait Posture. 2014 Feb; 39(2):784-8. http://doi.org/10.1016/i.gaitpost.2013.10.019 
14. Palacios-Navarro G, García-Magariño I, Ramos-Lorente P. A Kinect-Based System for Lower Limb Rehabilitation in Parkinson's Disease Patients: a Pilot Study. J Med Syst. 2015 Sep; 39(9):103. http://doi.org/10.1007/s10916-0150289-0

15. Alves Da Rocha P, McClelland J, Morris ME. Complementary physical therapies for movement disorders in Parkinson's disease: a systematic review. Eur J Phys Rehabil Med. 2015 Dec; 51(6):693-704. Epub 2015 Jul 3. PMID: 26138090.

16. Yang WC, Wang HK, Wu RM, Lo CS, Lin KH. Home-based virtual reality balance training and conventional balance training in Parkinson's disease: A randomized controlled trial. J Formos Med Assoc. 2016 Sep; 115(9):734-43. http://doi.org/10.1016/j.jfma.2015.07.012

17. Alves MLM, Mesquita BS, Morais WS, Leal JC, Satler CE, Dos Santos Mendes FA. Nintendo Wii' Versus Xbox Kinect ${ }^{\mathrm{TM}}$ for Assisting People With Parkinson's Disease. Percept Mot Skills. 2018 Jun; 125(3):546-65.

http://doi.org/10.1177/0031512518769204

18. Folstein MF, Folstein SE, McHugh PR. "Minimental state". A practical method for grading the cognitive state of patients for the clinician. J Psychiatr Res. 1975 Nov; 12(3):189-98. http://doi.org/10.1016/0022-3956(75)90026-6

19. Bertolucci PH, Brucki SM, Campacci SR, Juliano Y. O Mini-Exame do Estado Mental em uma população geral. Impacto da escolaridade [The Mini-Mental State Examination in a general population: impact of educational status]. Arq Neuropsiquiatr. 1994 Mar; 52(1):1-7. https://doi.org/10.1590/S0004-

282X1994000100001

20. Lourenço RA, Veras RP. Mini-Exame do Estado Mental: características psicométricas em idosos ambulatoriais [Mini-Mental State Examination: psychometric characteristics in elderly outpatients]. Rev Saude Publica. 2006 Aug; 40(4):712-9. DOI:

http://dx.doi.org/10.1590/s0034$\underline{89102006000500023}$

21. Bowden MG, Balasubramanian CK, Behrman AL, Kautz SA. Validation of a speed-based Colloq Vitae 2020 set-dez; 12(3): 1-9.

Artigo Open Access sob uma licença CC BY-NC-ND (http://creativecommons.org/licenses/by-nc-nd/4.0/) classification system using quantitative measures of walking performance poststroke. Neurorehabil Neural Repair. 2008 Nov-Dec; 22(6):672-5. http://doi.org/10.1177/1545968308318837

22. Bandeira Marina, Silva Mônia Aparecida da. Escala de Satisfação dos Pacientes com os Serviços de Saúde Mental (SATIS-BR): estudo de validação. J. bras. psiquiatr. 2012; 61( 3 ):12432.

\section{2}

23. Miranda PO, Souza OF, , Ferreira TF. Avaliação da satisfação dos pacientes e familiares em um serviço de saúde mental na cidade de Rio Branco, Acre. J. bras. psiquiatr. 2014 Dec; 63( 4 ): 332-40. https://doi.org/10.1590/00472085000000042

24. Wang B, Shen M, Wang YX, He ZW, Chi SQ, Yang $\mathrm{ZH}$. Effect of virtual reality on balance and gait ability in patients with Parkinson's disease: a systematic review and meta-analysis. Clin Rehabil. 2019 Jul; 33(7):1130-8. http://doi.org/10.1177/0269215519843174

25. Rochester L, Nieuwboer A, Baker K, Hetherington V, Willems AM, Kwakkel G et al. Walking speed during single and dual tasks in Parkinson's disease: which characteristics are important? Mov Disord. 2008 Dec 15; 23(16):2312-8.

http://doi.org/10.1002/mds.22219

26. Elbers RG, van Wegen EE, Verhoef J, Kwakkel $G$. Is gait speed a valid measure to predict community ambulation in patients with Parkinson's disease? J Rehabil Med. 2013 Apr; 45(4):370-5. http://doi.org/10.2340/16501977$\underline{1123}$

27. Parker N, Bugdayci D, Goksenoglu G, Demircioğlu DT, Kesiktas N, Ince N. Gait speed and related factors in Parkinson's disease. J Phys Ther Sci. 2015 Dec; 27(12):3675-9. http://doi.org/10.1589/ipts.27.3675

28. Baltadjieva R, Giladi N, Gruendlinger L, Peretz C, Hausdorff JM. Alterações marcadas no tempo de marcha e ritmicidade de pacientes com doença de Parkinson de novo . European Journal of Neuroscience 2006; 24: 1815-20. http://doi.org/10.1111/j.1460$\underline{\text { 9568.2006.05033.x }}$ 
29. Zettergren K, Franca J, Antunes M, Lavallee C. The effects of Nintendo Wii fit training on gait speed, balance, functional mobility and depression in one person with parkinson's disease. Med Health Sci J 2011; 9:18-24. http://doi.org/10.15208/ati.2011.11

30. Gonçalves GB, Leite MA, Orsini M, Pereira JS. Effects of using the nintendo wii fit plus platform in the sensorimotor training of gait disorders in Parkinson's disease. Neurol Int. 2014 Jan 17; 6(1):5048. http://doi.org/10.4081/ni.2014.5048

31. Kantorski LP, Jardim VR, Wetzel C, Olschowsky A, Schneider JF, Heck RM et al. Satisfação dos usuários dos centros de atenção psicossocial da região Sul do Brasil. Rev. Saúde Pública. 2009 Aug; 43(Suppl 1): 29-35. https://doi.org/10.1590/S0034-

$\underline{89102009000800006}$

32. Thiengo D, Fonseca D, Abelha L, Lovisi G. Satisfação de familiares com 0 atendimento oferecido por um Centro de Atenção Psicossocial Infantojuvenil (CAPSi) da cidade do Rio de Janeiro. Cadernos Saúde Coletiva. 2015; 23(3): 298-308. http://doi.org/10.1590/1414$\underline{462 \times 201500030172}$ 